\title{
Temporal effects of ruminal infusion of propionic acid on hepatic metabolism in cows in the postpartum period
}

\author{
Gabriela Maldini, ${ }^{*}$ Katherine M. Kennedy, and Michael S. Allen† \\ Department of Animal Science, Michigan State University, East Lansing 48824
}

\section{ABSTRACT}

A faster rate of infusion of propionic acid into the rumen of cows in the postpartum period increased meal size compared with a slower rate of infusion in a previous experiment. Because propionate is anaplerotic and stimulates oxidation of acetyl coenzyme A ( $\mathrm{CoA})$ in the liver, and hepatic oxidation has been linked to satiety, this result was opposite to our expected response. We then hypothesized that the faster rate of infusion might have saturated the pathway for propionate metabolism in hepatocytes resulting in lower first-pass extraction by the liver. Because we were measuring feeding behavior, we could not sample blood and liver tissue over time in that experiment. Therefore, to determine the temporal effects of propionic acid (PA) infusion on hepatic metabolism and plasma metabolites over the time course of a meal, we infused $1.25 \mathrm{~mol}$ of PA (2.5 L of $0.5 \mathrm{MPA})$ over $5 \mathrm{~min}$ (FST) or $15 \mathrm{~min}$ (SLW) into the rumen. We evaluated response to PA infusions both before feeding, when ruminal PA production by rumen microbes is lower and hepatic acetyl CoA concentration is greater, and $4 \mathrm{~h}$ after feeding, when PA production is greater and hepatic acetyl CoA concentration is lower. Blood and liver samples were collected before, and after 5, 15, and $30 \mathrm{~min}$ of infusion. Contrary to our hypothesis, the rate of $\mathrm{PA}$ infusion into the rumen did not affect plasma propionate concentration, indicating the FST effects on feeding behavior were not because of a limitation on propionate uptake by the liver. However, FST increased plasma glucose and insulin concentrations faster than SLW, resulting in a reduction in plasma nonesterified fatty acid concentration during the time frame of meals. Decreased plasma nonesterified fatty acid concentration during infusion likely decreased the supply of acetyl CoA for oxidation in the liver. The FST treatment also increased fumarate concentration

Received February 5, 2019.

Accepted June 23, 2019.

*Present address: 475 Saint Johns Church Road, Camp Hill, PA 17011.

†Corresponding author: allenm@msu.edu at 5 min after the initiation of infusion but did not affect oxaloacetate concentration compared with SLW, consistent with a limitation to propionate metabolism at that reaction. A metabolic bottleneck at the malate dehydrogenase reaction for FST compared with SLW would further contribute to a reduction in hepatic oxidation within the time frame of a meal, allowing greater meal size, consistent with the hepatic oxidation theory and our previous results.

Key words: feeding behavior, hepatic oxidation theory, satiety

\section{INTRODUCTION}

Propionic acid (PA) produced from ruminal fermentation of starch and other $\mathrm{OM}$ is the primary glucose precursor for ruminants (Lemosquet et al., 2009). For this reason, diets for lactating dairy cows are frequently formulated to increase starch concentration in an effort to increase the supply of glucose precursors to the cow. However, feeding diets that contain highly fermentable starch can decrease DMI (Allen, 2000; Sadri et al., 2012; Albornoz and Allen, 2018). Of the fuels produced during ruminal fermentation, PA is the fuel most likely to stimulate satiety and reduce feed intake in dairy cows (Allen, 2000).

Propionate is an obligatory anaplerotic metabolite for the tricarboxylic acid cycle (TCA), and its hypophagic effects are likely from its capacity to stimulate oxidation of hepatic acetyl CoA within the time frame of a meal (Allen et al., 2009). According to the hepatic oxidation theory, feeding behavior is controlled by signals transmitted to brain feeding centers through hepatic vagal afferents. Increased oxidation of fuels decreases the firing rate of the nerve, which is associated with satiety, whereas decreased oxidation of fuels increases the firing rate of the nerve, which is associated with hunger. The mechanism by which hepatic oxidation affects the firing rate of hepatic vagal afferents is yet to be determined, but research has consistently demonstrated the hypophagic effects of PA, that the signal is carried to brain feeding centers by hepatic vagal afferents (Allen et al., 2009), and that hypophagic effects of PA are related 
to its ability to stimulate hepatic oxidation (GualdrónDuarte and Allen, 2018).

In a previous experiment, we evaluated ruminal infusions of $1.25 \mathrm{~mol}$ of PA over $5 \mathrm{~min}$ (FST) and $15 \mathrm{~min}$ (SLW) at each meal (Maldini and Allen, 2018). We expected that FST would decrease meal size compared with SLW by stimulating hepatic oxidation and resulting in satiety sooner. Contrary to our hypothesis, we observed that FST increased meal size and decreased meal frequency when compared with SLW. We then hypothesized that the rapid supply of PA in the FST treatment saturated the pathway for propionate metabolism in the liver resulting in a by-pass of propionate. The extended interval between meals for FST compared with SLW suggested that a sustained propionate flux to the liver from the increased meal size and from propionate that escaped first-pass extraction extended hepatic oxidation over time, delaying hunger. Because we were evaluating feeding behavior, we were unable to sample blood or biopsy the liver over time during that experiment.

The objective of this experiment was to evaluate metabolic responses, including concentrations of plasma metabolites and hormones, and liver metabolites, to infusions of PA into the rumen of cows in the postpartum (PP) period using the same protocol and treatments of our previous experiment, before feeding and after feeding. We chose to evaluate treatment effects before and after feeding because propionate flux from the rumen to the liver is expected to be lower before feeding and higher after feeding. In addition, concentrations of nonesterified fatty acid (NEFA) in plasma and acetyl $\mathrm{CoA}$ in the liver are greater before feeding and lower after feeding (Piantoni et al., 2015). We hypothesized that FST would increase plasma propionate concentration following the start of infusion compared with SLW, characterizing the limitation on propionate uptake by the liver and subsequent bypass. Elevated concentrations of intermediates of propionate metabolism for FST compared with SLW would indicate bottlenecks to propionate metabolism. Because SLW was expected to result in greater anaplerosis of the TCA cycle over the time frame of meals because the propionate metabolism pathway would be less saturated, we hypothesized that SLW would stimulate acetyl CoA oxidation more quickly than FST, explaining the results in feeding behavior previously observed.

\section{MATERIALS AND METHODS}

\section{Animals, Housing, and Diets}

All experimental procedures were approved by the Institutional Animal Care and Use Committee at
Table 1. Ingredients and nutrient composition of the experimental diet (\% of dietary DM except for DM)

\begin{tabular}{lc}
\hline Item & $\%$ \\
\hline Ingredient & \\
Corn silage & 31.9 \\
Soybean meal & 19.2 \\
Ground corn & 19.1 \\
Alfalfa silage & 12.4 \\
Alfalfa hay & 9.7 \\
Soy hulls & 4.0 \\
Vitamin and mineral mix ${ }^{1}$ & 3.8 \\
Nutrient composition & \\
DM & 58.7 \\
OM & 91.3 \\
Starch & 24.9 \\
NDF & 29.5 \\
Forage NDF & 22.1 \\
CP & 17.2 \\
\hline
\end{tabular}

${ }^{1}$ Vitamin and mineral mix contained $25.6 \% \mathrm{NaCl}, 10.0 \% \mathrm{Ca}, 2.0 \% \mathrm{Mg}$, $2.0 \% \mathrm{P}, 30 \mathrm{mg} / \mathrm{kg}$ of $\mathrm{Co}, 506 \mathrm{mg} / \mathrm{kg}$ of $\mathrm{Cu}, 20 \mathrm{mg} / \mathrm{kg}$ of I, $2,220 \mathrm{mg} /$ $\mathrm{kg}$ of Fe, $2,080 \mathrm{mg} / \mathrm{kg}$ of Mn, $15 \mathrm{mg} / \mathrm{kg}$ of Se, $2,030 \mathrm{mg} / \mathrm{kg}$ of Zn, 300 $\mathrm{kIU} / \mathrm{kg}$ of vitamin A, $50 \mathrm{kIU} / \mathrm{kg}$ of vitamin $\mathrm{D}$, and $1,500 \mathrm{kIU} / \mathrm{kg}$ of vitamin $\mathrm{E}$.

Michigan State University, East Lansing. Cows were housed individually, and each cow was housed in the same tiestall for the duration of the experiment. Cows were fed once daily $(1200 \mathrm{~h})$ at $110 \%$ of expected intake and milked twice daily at 0530 and $1730 \mathrm{~h}$. All cows received a common experimental diet from parturition through the end of the experiment. The experimental diet (Table 1) was formulated to meet or exceed cow's estimated requirements according to the NRC (2001) and fed as a TMR. Before the initiation of the experiment, all animals were clipped over the 11,12 , and 13 intercostal areas from the backbone ventrally for approximately $30 \mathrm{~cm}$ to prepare for the liver biopsies.

\section{Experimental Design and Treatments}

Twelve multiparous Holstein cows $(\mathrm{n}=12,6 \pm 2 \mathrm{~d}$ $\mathrm{PP}$ ) in the PP period from the Michigan State University Dairy Teaching and Research Center were used in a crossover design experiment with double repeated measures. Cows $(n=2)$ with the closest range in days PP were selected for each block of this experiment. There was a total of 6 blocks, and each block was conducted over a 2-d period with 2 infusions per day. Cows within a block were randomly assigned to treatment sequence.

Treatments were infusion of $2.5 \mathrm{~L}$ of $0.5 \mathrm{M} \mathrm{PA}(1.25$ $\mathrm{mol})$ into the rumen over $5 \mathrm{~min}(0.25 \mathrm{~mol} / \mathrm{min}, \mathrm{FST})$ or over $15 \mathrm{~min}(0.08 \mathrm{~mol} / \mathrm{min}, \mathrm{SLW})$. Cows were infused twice per day, once before feeding (BF) and once after feeding (AF) through a nasogastric tube. The amount and length of time for infusions were selected based on our previous studies in which PA decreased DMI of cows in the PP period. Each cow within a block 
received the same treatment $\mathrm{BF}$ and $\mathrm{AF}$ on the same day (e.g., FST-BF, FST-AF on d 1, followed by SLW$\mathrm{BF}, \mathrm{SLW}-\mathrm{AF}$ on $\mathrm{d} 2$ or the reverse).

\section{Sample Collection and Analysis}

Samples of all dietary ingredients were collected daily and composited to characterize the experimental diet. Feed ingredients were dried in a $55^{\circ} \mathrm{C}$ forced-air oven for $72 \mathrm{~h}$ and analyzed for DM concentration. Samples were ground with a Wiley mill (1-mm screen; Arthur H. Thomas, Philadelphia, PA) and analyzed for ash, NDF, CP, and starch as described by Maldini and Allen (2018).

Feed access was blocked $4 \mathrm{~h}$ before feeding when cows were cleaned in preparation for the biopsies. Briefly, the incision site was cleaned with $7.5 \%$ povidone-iodine followed by $70 \%$ ethanol and anesthetized locally with lidocaine $2 \%\left(20 \mathrm{~cm}^{3}\right)$. The effectiveness of the local anesthesia was tested before collection of tissue, and if the cow reacted to stimulus after $10 \mathrm{~min}$ of the first anesthesia, up to $10 \mathrm{~cm}^{3}$ of additional lidocaine was administered. Cows were then restrained in a chute to pass the nasogastric tube into the rumen. A clear, long flexible veterinary stomach tube $(12.7 \mathrm{~mm} \times 274.3 \mathrm{~cm})$ was placed in warm water for 5 min previous to insertion to increase flexibility. We verified that the tubing was in the rumen by blowing air in the tube and listening for burbling noises, and that rumen gas was escaping the rumen through the tube. Once placed, the tube was attached to the halter and cows were returned to their stalls. In the stalls, the nasogastric tubing was connected to a peristaltic pump (FPU401, Omegaflex Peristaltic Pump, Norwalk, CT). Tubes were removed immediately after infusions were finished.

Approximately $1 \mathrm{~h}$ before the estimated feeding time, blood and liver tissues were sampled to determine baselines values immediately before infusion to each animal. Infusion was then initiated and consecutive blood and liver samples were taken during and after infusion at 5,15 , and $30 \mathrm{~min}$ following the initiation of infusion. Blood was sampled from coccygeal vessels and collected into 4 evacuated tubes, 3 containing $\mathrm{K}_{2}$ EDTA and 1 containing potassium oxalate with $\mathrm{NaF}$ as a glycolytic inhibitor and kept on ice until the last sample was harvested. Blood was immediately centrifuged at 1,700 $\times$ $g$ for $15 \mathrm{~min}$ at $5^{\circ} \mathrm{C}$. Plasma was harvested, and 200 $\mu \mathrm{L}$ of benzamidine $(0.25 \mathrm{M})$ was added to $1,000 \mu \mathrm{L}$ of plasma as a protease inhibitor for glucagon analysis. All samples were immediately frozen and kept at $-20^{\circ} \mathrm{C}$ until analysis.

Liver tissue was biopsied according to Bradford and Allen (2005) as modified by Ferreira et al. (1996). At each sampling time, a 14-gauge biopsy needle was inserted into the intercostal space between the 11th and 12 th ribs on a line between the olecranon and the tuber coxae on the right side. Eight samples of approximately $20 \mathrm{mg}$ each were collected, snap frozen in liquid nitrogen, and stored at $-80^{\circ} \mathrm{C}$ until analysis. Cows were monitored daily following biopsies, and no health complications were observed during the experiment.

After the last sampling BF, cows were allowed access to feed. Cows were allowed to eat for $4 \mathrm{~h}$, until access to feed was prevented for the second time for the $\mathrm{AF}$ collection. Cows were again cleaned and anesthetized. Nasogastric tubes were placed and samples were collected for baseline. Infusion was initiated with the same treatment as in the morning, and samples were collected at 5, 15, and $30 \mathrm{~min}$ after initiation of infusion. After the last sample, cows were immediately allowed to eat until the next sampling day.

Commercial kits were used to analyze plasma concentrations of NEFA (NEFA HR kit, Wako Chemicals USA, Richmond, VA), BHB (Stanbio Laboratory, Boerne, TX), and glucose (glucose oxidase, Sigma Chemical Co., St. Louis, MO), and samples were analyzed colorimetrically with a microplate reader (SpectraMax Plus 384, Molecular Devices Corp., Sunnyvale, CA) to determine concentrations of metabolites. Plasma glucagon concentration was determined using RIA kit (kit no. GL-32K, Linco Research Inc., St. Charles, MO), and plasma insulin concentration was determined using an ELISA kit (Mercodia, Uppsala, Sweden). Plasma propionate, acetate, butyrate, and organic acid concentrations were analyzed by HPLC (Waters Corp., Milford, MA) as described by Gualdrón-Duarte and Allen (2017).

Liver metabolites related to hepatic metabolism, including succinate, fumarate, malate, oxaloacetate, citrate, $\alpha$-ketoglutarate, glutamate, pyruvate, lactate, aspartate, 3-hydroxybutyrate, glucose, and $\gamma$-aminobutyric acid (GABA), were analyzed using GC-MS, and CoA, acetyl CoA, propionyl CoA, succinyl CoA, and methylmalonyl CoA were analyzed using liquid chromatography-MS/MS (LC-MS/MS). Approximately $40 \mathrm{mg}$ of frozen tissue was weighed into clean, flat-bottom 2-mL centrifuge tubes and stored on dry ice. Exact weight of tissue was recorded and used to correct samples to a per milligram wet weight basis. Cold $1 \%$ formic acid in $70 \%$ acetonitrile: $30 \% \mathrm{H}_{2} \mathrm{O}$ was added $(500 \mu \mathrm{L})$ to each tube and immediately homogenized using a Polytron (Kinematica, Luzern, Switzerland) homogenizer. After homogenization, samples were centrifuged at $5,000 \times g$ for $10 \mathrm{~min}$ at $4^{\circ} \mathrm{C}$. Aliquots of supernatant $(100 \mu \mathrm{L})$ were transferred to clean centrifuge tubes for analysis and stored at $-80^{\circ} \mathrm{C}$. All 
samples for GC-MS and LC-MS/MS were evaporated to dryness using a Savant SC110 SpeedVac Concentrator (Savant Instruments Inc., Holbrook, NY).

$G C$-MS. Samples evaporated to dryness were derivatized by methoximation and trimethylsilylation as modified by Kind et al. (2009). Briefly, $30 \mu \mathrm{L}$ of O-methylhydroxylamine reagent solution $(40 \mathrm{mg} / 1 \mathrm{~mL}$ of pyridine; Sigma Aldrich, St. Louis, MO) was added to each sample and vortexed for $30 \mathrm{~s}$ for homogenization. Samples were then shaken for $3 \mathrm{~h}$ at $60^{\circ} \mathrm{C}$ in a Forma Orbital Shaker Model 420 (Thermo Fisher Scientific, Waltham, MA). An additional $90 \mu \mathrm{L}$ of either $N$-tert-butyldimethylsilyl- $N$-methyltrifluoroacetamide (for pyruvate, citrate, succinate, fumarate, malate, BHB, lactate, phosphate, aspartate, and glutamate) or N,O-Bis(trimethylsilyl)trifluoroacetamide (for keto glutarate, oxaloacetate, glucose, and GABA) was added to each sample, vortexed for $10 \mathrm{~s}$, and then shaken for an additional $30 \mathrm{~min}$ at $37^{\circ} \mathrm{C}$. Samples were then centrifuged at $5,000 \times g$ for $10 \mathrm{~min}$ each at $22^{\circ} \mathrm{C}$. The supernatant was transferred into a $250-\mu \mathrm{L}$ glass insert within a 2-mL amber autosampler vial (Thermo Fisher Scientific). Vials were stored in a desiccator in the dark until use for GC-MS within $24 \mathrm{~h}$ of derivatization.

Samples were analyzed with GC-MS as described by Kennedy and Allen (2019). Analysis of peaks was performed with MassLynx Mass Spectrometry Software (version 4.1, Waters Corp.). Peaks were identified and quantified by comparison with standards.

$L C-M S / M S$. Samples were analyzed according to Gotoh et al. (2015), with modifications by Kennedy and Allen (2019). Analysis of peaks was performed with MassLynx Mass Spectrometry Software (version 4.1, Waters Corp.). Peaks were identified and quantified by comparison with standards. Samples evaporated to dryness were resuspended in $400 \mathrm{~m} M$ ammonium formate with $10 \mathrm{~m} M$ Tris (2-carboxyethyl) phosphine hydrochloride (Sigma Aldrich). Samples were centrifuged at $5,000 \times g$ for $10 \mathrm{~min}$ at $4^{\circ} \mathrm{C}$, transferred to a new clean vial, and centrifuged a second time at $5,000 \times g$ for 10 min at $4^{\circ} \mathrm{C}$. The supernatant was then transferred into a 250- $\mu \mathrm{L}$ glass insert within a $2-\mathrm{mL}$ amber autosampler vial (Thermo Fisher Scientific) for analysis on LC-MS/ MS.

\section{Statistical Analysis}

Before statistical analysis, Box-Cox transformation analyses were applied to each response variable to determine necessary transformations on the data using JMP Pro (version 13.2, 2016; SAS Institute Inc., Cary, $\mathrm{NC}$ ). Data were then analyzed using the MIXED procedure of SAS (version 9.4, 2013; SAS Institute Inc.) with double repeated measures. Hepatic and plasma metabolites and hormones were analyzed using the following model:

$$
\begin{gathered}
Y_{i j k l m n}=\mu+C_{i}\left(B_{j}\right)+P_{k}\left(B_{j}\right)+B_{j}+T_{l}+S_{m}+F_{n} \\
+T_{l} S_{m}+T_{l} F_{n}+F_{n} S_{m}+T_{l} F_{n} S_{m}+e_{i j k l m n}
\end{gathered}
$$

where $Y_{i j k l m n}=$ the response variable; $\mu=$ overall mean; $C_{i}\left(B_{j}\right)=$ random effect of cow $i$ nested within block $j ; P_{k}\left(B_{j}\right)=$ random effect of period $k$ nested within block $j ; B_{j}=$ random effect block; $T_{l}=$ fixed effect of treatment (rate of infusion: FST, SLW); $S_{m}=$ fixed effect of sampling time (before infusion and 5, 15, and 30 min after infusion); $F_{n}=$ fixed effect of time of infusion relative to feeding ( $\mathrm{BF}, \mathrm{AF}) ; T_{l} S_{m}=$ interaction of treatment (rate of infusion) and sampling time; $T_{l} F_{n}$ = interaction of treatment (rate of infusion) and time of infusion relative to feeding; $F_{n} S_{m}=$ interaction of time of infusion relative to feeding and sampling time; $T_{l} F_{n} S_{m}=$ interaction of treatment (rate of infusion), time of infusion relative to feeding, and sampling time; and $e_{i j k l m n}=$ residual. Time of infusion relative to feeding was the macro repeated measure, and sampling time was the micro repeated measure. The covariance structure was unstructured @ compound symmetry for all variables. Paired differences were determined using the either the diff or slice option in PROC MIXED.

Treatment effects were declared at $P \leq 0.05$ and tendencies for treatment effects were declared at $P \leq 0.10$. Interactions were declared significant at $P \leq 0.10$ and tendencies for interactions were declared at $P \leq 0.15$. Data transformed are noted in the tables and figures along with the transformation used for statistical analysis. All transformed data has been back-transformed for interpretation.

\section{RESULTS}

\section{Blood Metabolites and Hormones}

Rate of infusion and time of infusion relative to feeding interacted with time to affect plasma propionate concentration ( $P=0.06$; Table 2, Figure 1a). Plasma propionate concentration increased for SLW before feeding, peaking at $15 \mathrm{~min}$ of infusion $(P<0.05$, LSM diff $)$ and tended to be greater at $30 \mathrm{~min}(P=0.06$, LSM diff) compared with the preinfusion sample. In contrast, plasma propionate concentration did not increase for FST before feeding, and when compared with the preinfusion sample, FST-BF tended to decrease plasma propionate by 30 min after the start of infusion $(P=0.06$, LSM diff). Plasma propionate concentration did not differ from the preinfusion sample for either treatment when PA was infused after feeding. 
Table 2. Concentration of plasma metabolites and hormones over the first $30 \mathrm{~min}$ after infusion of 1.25 mol of propionic acid over 5 min (FST) or $15 \mathrm{~min}(\mathrm{SLW})$ before feeding $(\mathrm{BF})$ and $4 \mathrm{~h}$ after feeding $(\mathrm{AF})$

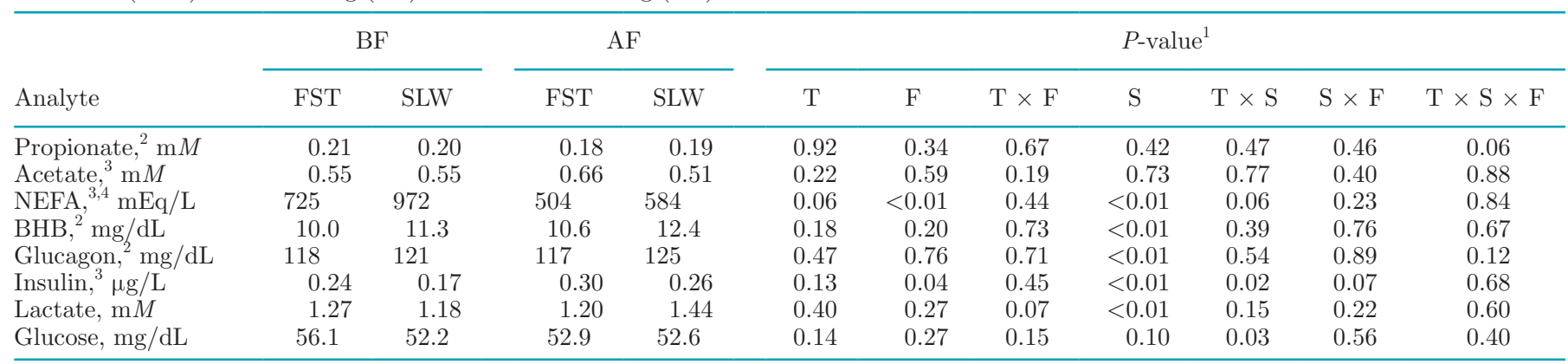

${ }^{1} \mathrm{~T}=$ treatment $(\mathrm{FST}, \mathrm{SLW}), \mathrm{F}=$ time of infusion relative to feeding $(\mathrm{BF}, \mathrm{AF}), \mathrm{S}=$ sampling time (before infusion and after 5,15, and 30 min from the initiation of infusion).

${ }^{2}$ Data were analyzed after square root transformation and back transformed for interpretation.

${ }^{3}$ Data were analyzed after log transformation and back transformed for interpretation.

${ }^{4} \mathrm{NEFA}=$ nonesterified fatty acids.

Rate of infusion interacted with sampling time to affect plasma glucose concentration $(P=0.03$; Table 2) with FST increasing glucose concentration until 15 min but SLW decreasing glucose concentration at 5 min with similar concentrations for both treatments by 30 min (Figure 1b). Plasma glucose concentration also tended to be affected by an interaction of rate of infusion and time of infusion relative to feeding $(P=0.15)$ with a greater concentration for FST before feeding than FST after feeding or SLW before or after feeding.

Rate of infusion and time of infusion relative to feeding tended to interact with sampling time to affect plasma glucagon concentration $(P=0.12$, Figure 1c). Plasma glucagon concentration did not differ by treatment at any individual sampling time $(P>0.15$, LSM diff) either BF or AF; however, when compared with the preinfusion sample, SLW increased glucagon concentration at $15 \mathrm{~min} \mathrm{BF}$, whereas no change in plasma glucagon existed for FST. After feeding, plasma glucagon concentration did not differ from the preinfusion sample for either treatment.

Rate of infusion and sampling time interacted to affect plasma insulin concentration $(P=0.02$; Figure 1d). The FST treatment increased plasma insulin concentration compared with SLW as soon as $5 \mathrm{~min}$ from the start of infusion $(P<0.01$, slice test), but concentrations were similar by $30 \mathrm{~min}(P>0.10$, slice test). Plasma insulin concentration was greater $\mathrm{AF}$ than $\mathrm{BF}$ (0.28 vs. $0.21 \mu \mathrm{g} / \mathrm{L}, P=0.04)$ and varied from 0.24 to $0.013 \mu \mathrm{g} / \mathrm{L} \mathrm{BF}$ and from 0.29 to $0.17 \mathrm{AF}$ (sampling time and time relative to feeding interaction, $P=0.07$ ).

The FST treatment tended to decrease plasma NEFA concentration $(P=0.06)$, and the decrease was more rapid compared with SLW $(P=0.06$; Figure 1e). Baseline plasma samples did not differ $(P>0.10$, slice test $)$, but after 5 min of the infusion initiation, FST reduced plasma NEFA concentration $(P<0.01$, slice test $)$ compared with SLW and further reduced it by $15 \mathrm{~min}(P<$ 0.05 , slice test). Plasma NEFA concentration was lower AF compared with BF (544 vs. $849 \mathrm{mEq} / \mathrm{L} ; P<0.01$ ).

Compared with SLW, FST treatment increased plasma lactate concentration $\mathrm{BF}(1.27$ vs. $1.18 \mathrm{mM})$ but decreased it AF (1.20 vs. $1.44 \mathrm{mM} P=0.07)$. Infusion treatments tended to interact with time to affect plasma lactate concentration $(P=0.15$; Figure 1f). No treatment differences existed from baseline until 15 min; however, at 30 min SLW tended to increase plasma lactate compared with FST $(P=0.08$, LSM diff). Plasma acetate and BHB (Figure $1 \mathrm{~g}$ ) were not affected by treatment or time of infusion relative to feeding.

\section{Hepatic Metabolites}

No main effects of treatment were detected for any of the metabolites measured, and no treatment $\times$ sampling time interactions were detected with the exception of fumarate and lactate (Table 3). Treatments tended to interact with time to affect fumarate concentration $(P$ $=0.12$, Figure 2a); FST significantly increased hepatic fumarate concentration at 5 min compared with SLW $(P=0.05$, slice test). Fumarate concentrations were greater $\mathrm{AF}$ compared with $\mathrm{BF}$ (37.7 vs. $31.2 \mu \mathrm{mol} /$ $\mathrm{mg}, P=0.05)$. Lactate concentrations were greater $\mathrm{AF}$ compared with $\mathrm{BF}(P<0.01)$, but treatments tended to interact with time of infusion relative to feeding and sampling time $(P=0.12$; Figure $2 \mathrm{~b})$.

Most metabolites decreased or tended to decrease AF compared with $\mathrm{BF}$ including propionyl CoA (0.53 vs. $1.27 \mu \mathrm{mol} / \mathrm{mg} ; P<0.01)$, methylmalonyl CoA (0.22 vs. $0.37 \mu \mathrm{mol} / \mathrm{mg} ; P=0.05)$, succinyl CoA (3.36 vs. 5.53 $\mu \mathrm{mol} / \mathrm{mg} ; P=0.02$ ), succinate (35 vs. $58 \mu \mathrm{mol} / \mathrm{mg}$; 

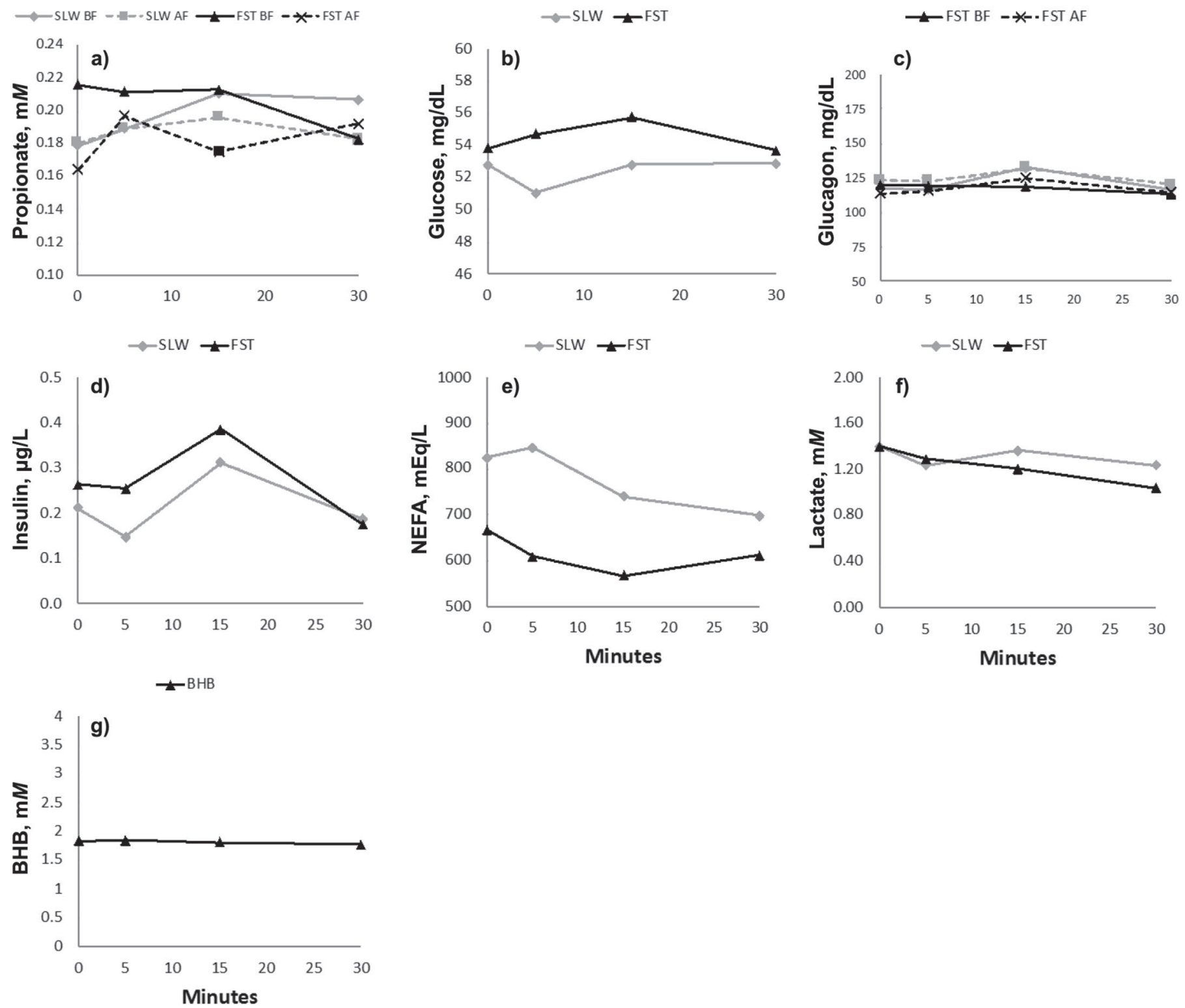

Figure 1. Plasma propionate (a), glucose (b), glucagon (c), insulin (d), nonesterified fatty acids (NEFA; e), lactate (f) and BHB (g) over the first $30 \mathrm{~min}$ after infusion of $1.25 \mathrm{~mol}$ of propionic acid over $5 \mathrm{~min}$ (SLW) or $15 \mathrm{~min}$ (FST). Data for propionate and glucagon were analyzed after square root transformation and back transformed for interpretation. Data for insulin, NEFA, and lactate were analyzed after log transformation and back transformed for interpretation. $\mathrm{BF}=$ before feeding; $\mathrm{AF}=$ after feeding.

$P=0.01$ ), fumarate (31 vs. $38 \mu \mathrm{mol} / \mathrm{mg} ; P=0.05)$, malate (72 vs. $114 \mu \mathrm{mol} / \mathrm{mg} ; P=0.02)$, GABA $(337$ vs. $284 \mu \mathrm{mol} / \mathrm{mg} ; P=0.06)$, glutamate (1,501 vs. 1,061 $\mu \mathrm{mol} / \mathrm{mg} ; P=0.03)$, and $\mathrm{CoA}(3.68$ vs. $6.32 \mu \mathrm{mol} / \mathrm{mg}$; $P=0.03$ ). However, pyruvate $(69$ vs. $79 \mu \mathrm{mol} / \mathrm{mg} ; P$ $=0.03)$ and lactate $(981$ vs. $1,550 \mu \mathrm{mol} / \mathrm{mg} ; P<0.01)$ increased AF compared with BF (Table 3).

Rate of infusion interacted with time of infusion relative to feeding to affect acetyl CoA $(P=0.08)$, and inorganic phosphate $(P=0.09)$ and tended to interact for glucose $(P=0.11)$. The FST treatment decreased acetyl CoA BF (4.16 vs. $4.46 \mu \mathrm{mol} / \mathrm{mg}$ ) and increased acetyl CoA AF (5.71 vs. $3.45 \mu \mathrm{mol} / \mathrm{mg}$ ) compared with SLW. The SLW treatment increased glucose (1,877 vs. $1,762 \mu \mathrm{mol} / \mathrm{mg}$ ) and inorganic phosphate (5,658 vs. $5,308 \mu \mathrm{mol} / \mathrm{mg}$ ) BF but decreased glucose (1,304 vs. $1,725 \mu \mathrm{mol} / \mathrm{mg}$ ) and inorganic phosphate (4,555 vs. $5,421 \mu \mathrm{mol} / \mathrm{mg}$ ) AF compared with FST.

Time of infusion relative to feeding and sampling time interacted to affect $\mathrm{BHB}(P=0.03$, Figure $2 \mathrm{c})$, 
Table 3. Concentration of hepatic metabolites $(\mu \mathrm{mol} / \mathrm{mg})$ related to propionate metabolism over the first 30 min after infusion of $1.25 \mathrm{~mol}$ of propionic acid over $5 \mathrm{~min}$ (FST) or $15 \mathrm{~min}$ (SLW) before feeding (BF) and $4 \mathrm{~h}$ after feeding (AF)

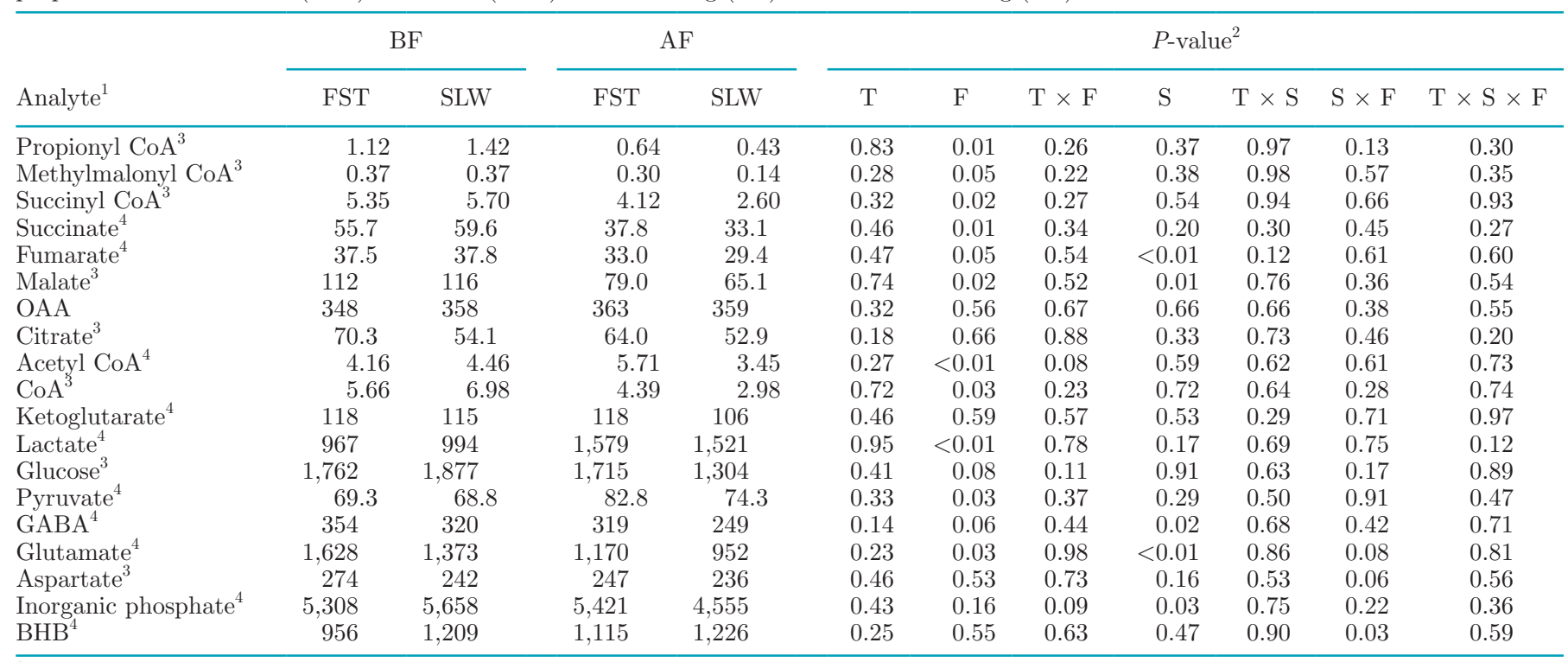

${ }^{1} \mathrm{OAA}=$ oxaloacetate; $\mathrm{GABA}=\gamma$-aminobutyric acid.

${ }^{2} \mathrm{~T}=$ treatment $(\mathrm{FST}, \mathrm{SLW}), \mathrm{F}=$ time of infusion relative to feeding (BF, AF), $\mathrm{S}=$ sampling time (before infusion and after 5,15 , and 30 min from the initiation of infusion).

${ }^{3}$ Data were analyzed after log transformation and back transformed for interpretation.

${ }^{4}$ Data were analyzed after square root transformation and back transformed for interpretation.

glutamate $(P=0.08$, Figure $2 \mathrm{~d})$, and aspartate $(P=$ 0.06, Figure 2e). The BHB increased from 994 to 1,115 $\mu \mathrm{mol} / \mathrm{mg}$ from baseline to $30 \mathrm{~min} \mathrm{BF}$, whereas it decreased from 1,303 to $1,009 \mu \mathrm{mol} / \mathrm{mg}$ AF. Glutamate decreased from 1,749 to $1,455 \mu \mathrm{mol} / \mathrm{mg} \mathrm{BF}$ and from 1,533 to $670 \mu \mathrm{mol} / \mathrm{mg}$ AF. Aspartate varied from 256 to $265 \mu \mathrm{mol} / \mathrm{mg} \mathrm{BF}$ and decreased from 271 to 198 $\mu \mathrm{mol} / \mathrm{mg} \mathrm{AF}$. Time of infusion relative to feeding and sampling time tended to affect propionyl CoA (Figure 2f), and concentrations varied from 1,685 to $778 \mu \mathrm{mol} /$ $\mathrm{mg} \mathrm{BF}$ and from 781 to $419 \mu \mathrm{mol} / \mathrm{mg} \mathrm{AF}$.

Sampling time effects for malate, inorganic phosphate, and GABA are presented in Figure 2. Malate concentration increased slightly at $5 \mathrm{~min}$ and decreased at 15 and 30 min after infusion independent of treatment $(P=0.01$, Figure $2 \mathrm{~g})$, whereas inorganic phosphate $(P=0.03$, Figure $2 \mathrm{~h})$ and $\operatorname{GABA}(P=0.02$, Figure 2i) decreased over time.

\section{DISCUSSION}

Propionate extracted from the blood by the liver can stimulate oxidation of acetyl CoA in the TCA cycle contributing to satiety according to the hepatic oxidation theory (Allen et al., 2009). However, fractional hepatic extraction of propionate can be decreased when $\mathrm{PA}$ is infused intraruminally in sheep at $1.78 \mathrm{mmol} / \mathrm{h}$ per kilogram of BW for $4 \mathrm{~h}$ (Berthelot et al., 2002) and mesenterically in cows at $1.2 \mathrm{mmol} / \mathrm{h}$ per kilogram of BW (Baird et al., 1980). As a result, propionate would be expected to increase in the peripheral circulation. Oba and Allen (2003) reported that plasma propionate concentration increased linearly from 0.19 to $0.34 \mathrm{mM}$ when propionate was infused intraruminally from 0 to $1.3 \mathrm{~mol} / \mathrm{h}$ for cows in the PP period.

In a previous experiment, we observed an increase in meal size and extended time between meals by FST, reducing meal frequency of cows in the PP period (Maldini and Allen, 2018), and we hypothesized that FST saturated the pathway for propionate metabolism resulting in a lower first-pass extraction by the liver compared with SLW. However, the results of this experiment do not support our hypothesis. On the contrary, SLW increased and FST decreased plasma propionate concentration over time BF with no consistent difference $\mathrm{AF}$. We infused PA for a maximum of $15 \mathrm{~min}$, whereas most of the research cited infused PA continuously for at least $4 \mathrm{~h}$ (up to $18 \mathrm{~h}$ ). The lack of treatment effects in our experiment suggests that a more prolonged and more continuous infusion of PA may be required to saturate hepatic propionate metabolism. Because our goal was to evaluate metabolic changes within a meal, we restricted our infusions and sampling to an average meal length, and therefore, the cumulative effects of PA continuous infusion on metabolism were probably not captured. 
The lack of treatment effects and their interactions with sampling time for plasma propionate concentration as well as concentrations of propionyl CoA, methylmalonyl CoA, succinyl CoA, and succinate in the liver are consistent with the idea that efficiency of extraction of propionate by the liver from the blood was not affected by treatment. However, whereas significant differences between treatments were not detected overall, plotting concentrations of metabolites over time for individual cows indicated that the site of potential bottlenecks at which individual metabolites of propionate metabolism were elevated varied among cows. The only potential bottleneck identified among cows was at the conversion of fumarate to malate, indicated by an accumulation
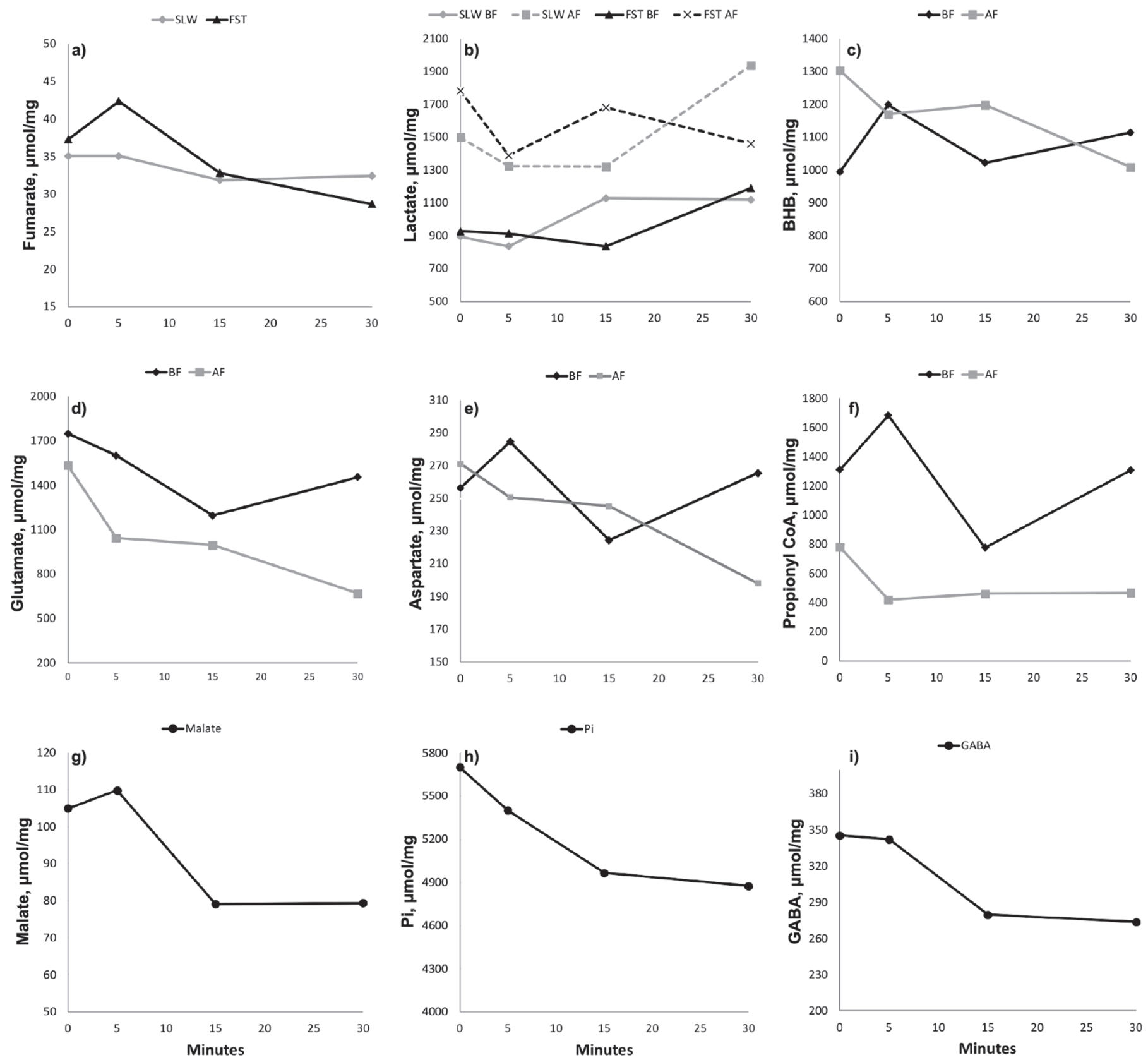

Figure 2. Fumarate (a), lactate (b), BHB (c), glutamate (d), aspartate (e), propionyl CoA (f), malate (g), inorganic phosphate (Pi; h), and $\gamma$-aminobutyric acid (GABA; i) concentration in liver tissue over the first 30 min after infusion of 1.25 mol of propionic acid over 5 min (SLW) or $15 \mathrm{~min}$ (FST). Data for fumarate, lactate, BHB, glutamate, Pi, and GABA were analyzed after square root transformation and back transformed for interpretation. Data for aspartate and malate were analyzed after log transformation and back transformed for interpretation. $\mathrm{BF}=$ before feeding; $\mathrm{AF}=$ after feeding. 
of fumarate over time for FST compared with SLW. We expected that the malate dehydrogenase reaction might be a limitation to propionate metabolism in the TCA cycle when $\mathrm{NADH} / \mathrm{NAD}^{+}$is elevated, resulting in elevated malate concentration in the mitochondria. Whereas malate concentration was not affected by treatment, we measured metabolite concentrations in whole liver tissue. Mitochondrial malate concentration might have increased causing the increase observed for fumarate, its upstream precursor, for FST compared with SLW with no overall increase for malate concentration in whole liver tissue.

Propionate enters the TCA cycle through succinate, thus providing carbons that can either remain within the TCA cycle or can be extracted from the cycle for gluconeogenesis (White, 2015). The more rapid increase in plasma glucose for FST compared with SLW with no effect of treatment on oxaloacetate or citrate is consistent with the increased flow of propionate carbon from FST being exported to the cytosol rather than stimulating oxidation of acetyl CoA. The rapid increase in plasma glucose concentration for FST compared with SLW was not related to changes in plasma glucagon concentration, which was increased by SLW but not FST.

Rate of oxidation in hepatocytes is dependent on the number of TCA intermediates, which is determined by the balance between anaplerosis and cataplerosis, the availability of acetyl CoA for the citrate synthase reaction, and the speed at which the cycle spins, affected by enzyme concentration and activity (Allen, 2014). The primary source of acetyl $\mathrm{CoA}$ is from mitochondrial $\beta$-oxidation of NEFA extracted from the blood by the liver (Allen et al., 2009; White, 2015).

Hypophagic effects of propionate infusions were greater with elevated hepatic acetyl CoA concentrations in early-lactation cows (Stocks and Allen, 2012, 2013), likely because of increased anaplerosis and oxidation in the TCA cycle. Hepatic concentration of acetyl CoA is dependent on the balance between its supply and its disappearance from oxidation in the TCA cycle or export from the liver as ketone bodies and acetate. No temporal effects of treatment existed within infusions on hepatic acetyl CoA and BHB concentrations, or on plasma BHB and acetate concentrations. However, plasma NEFA concentration decreased faster for FST compared with SLW, which likely reduced the supply of acetyl CoA in the TCA cycle. Therefore, hepatic oxidation might have been slower for FST compared with SLW because acetyl CoA concentration was not reduced by FST during the time frame of meals. That would explain how FST allowed for greater meal size in our previous experiment (Maldini and Allen, 2018). Consistent with this, Piantoni et al. (2015) showed a positive relationship between the extent of reduction in plasma NEFA concentration and DMI during the first $4 \mathrm{~h}$ after feeding for cows in the PP period and that the reduction in plasma NEFA concentration and the reduction in hepatic acetyl CoA concentration were linearly related.

The reduction in concentrations of hepatic metabolites in the pathway from propionate to malate AF compared with BF was not expected because of the greater anaplerosis from propionate and other metabolites to the liver AF compared with BF. Because concentration is a function of the balance between anaplerosis and cataplerosis, and anaplerosis was likely increased AF, their reduction in concentration indicates that cataplerosis was also increased AF, likely from increased gluconeogenesis. Whereas plasma glucose concentration was not greater $\mathrm{AF}$ compared with $\mathrm{BF}$, propionate is a potent insulin secretagogue in ruminants (Leuvenink at al., 1997) and greater insulin concentration likely increased glucose clearance from the blood.

Effects of treatment on neurotransmitters produced and potentially released by hepatocytes were investigated as a potential mechanism for the communication between hepatocytes and hepatic vagal afferents as well as differences between treatments on feeding behavior. According to the hepatic oxidation theory, inhibition of hepatic vagal afferents causes satiety and excitation stimulates hunger (Allen et al., 2009). $\gamma$-Aminobutyric acid is an inhibitory neurotransmitter, whereas glutamate and aspartate are excitatory neurotransmitters and all are produced by hepatic metabolism. However, no effects of treatment existed nor interactions with sampling time on concentration of any of these neurotransmitters, and both glutamate and GABA decreased over time similarly across treatments with little change in aspartate.

\section{CONCLUSIONS}

Whereas we cannot discern the pattern of oxidation of acetyl CoA from our data, the more rapid increase in plasma glucose and insulin concentrations, and more rapid reduction in plasma NEFA concentration for FST compared with SLW, likely decreased supply of acetyl CoA for oxidation in the TCA cycle, which might have allowed greater meal size for the FST treatment compared with SLW observed in our previous experiments. Increased meal size would be expected to extend the time between meals, decreasing meal frequency. To our knowledge, the current experiment is the first to attempt to evaluate the effects of temporal supply of propionate within a meal on hepatic metabolism to explain feeding behavior. These results will help our understanding of the complex mechanisms controlling 
feed intake in dairy cows. Further research involving the metabolism of propionate relative to meals and its relation to hepatic energy status is needed to better understand metabolic control of intake in ruminants.

\section{ACKNOWLEDGMENTS}

We acknowledge funding for this project by USDA National Research Initiative Competitive Grant no. 2014-67015-21708 to M. S. Allen and from Coordenação de Aperfeiçoamento de Pessoal de Nível Superior (CAPES)-Brazilian Federal Agency for Support and Evaluation of Graduate Education within the Ministry of Education of Brazil (Brasília, Brazil) to G. Maldini while conducting this research. We thank D. G. Main, L. G. Duarte, and R. Albornoz (all from Michigan State University, Lansing) for their assistance and Kemin Animal Nutrition and Health (Des Moines, IA) for supplying the propionic acid.

\section{REFERENCES}

Albornoz, R. I., and M. S. Allen. 2018. Highly fermentable starch at different diet starch concentrations decreased feed intake and milk yield of cows in the early postpartum period. J. Dairy Sci. 101:8902-8915. https://doi.org/10.3168/jds.2018-14843.

Allen, M. S. 2000. Effects of diet on short-term regulation of feed intake by lactating dairy cattle. J. Dairy Sci. 83:1598-1624. https:// doi.org/10.3168/jds.S0022-0302(00)75030-2.

Allen, M. S. 2014. Drives and limits to feed intake in ruminants. Anim. Prod. Sci. 54:1513-1524. https://doi.org/10.1071/AN14478.

Allen, M. S., B. J. Bradford, and M. Oba. 2009. Board-invited review: The hepatic oxidation theory of the control of feed intake and its application to ruminants. J. Anim. Sci. 87:3317-3334. https://doi .org/10.2527/jas.2009-1779.

Baird, G. D., M. A. Lomax, H. W. Symonds, and S. R. Shaw. 1980. Net hepatic and splanchnic metabolism of lactate, pyruvate and propionate in dairy cows in vivo in relation to lactation and nutrient supply. Biochem. J. 186:47-57. https://doi.org/10.1042/ bj1860047.

Berthelot, V., S. G. Pierzynowski, D. Sauvant, and N. B. Kristensen. 2002. Hepatic metabolism of propionate and methylmalonate in growing lambs. Livest. Prod. Sci. 74:33-43. https://doi.org/10 .1016/S0301-6226(01)00281-0.

Bradford, B. J., and M. S. Allen. 2005. Phlorizin administration increases hepatic gluconeogenic enzyme mRNA abundance but not feed intake in late-lactation dairy cows. J. Nutr. 135:2206-2211. https://doi.org/10.1093/jn/135.9.2206.

Ferreira, A. V., H. J. Van der Merwe, and S. C. Slippers. 1996. A technique for obtaining liver biopsies from mature sheep. Small Rumin. Res. 22:89-92. https://doi.org/10.1016/0921-4488(96)00858-9.

Gotoh, K., Y. Nakajima, G. Tajima, Y. Hotta, T. Kataoka, Y. Kawade, N. Sugiyama, T. Ito, K. Kimura, and Y. Maeda. 2015. Assay for methylmalonyl coenzyme A mutase activity based on determi- nation of succinyl coenzyme A by ultrahigh-performance liquid chromatography tandem mass spectrometry. Anal. Bioanal. Chem. 407:5281-5286. https://doi.org/10.1007/s00216-015-8753-8.

Gualdrón-Duarte, L. B., and M. S. Allen. 2017. Increased anaplerosis of the tricarboxylic acid cycle decreased meal size and energy intake of cows in the postpartum period. J. Dairy Sci. 100:44254434. https://doi.org/10.3168/jds.2016-12104.

Gualdrón-Duarte, L. B., and M. S. Allen. 2018. Fuels derived from starch digestion have different effects on energy intake and metabolic responses of cows in the postpartum period. J. Dairy Sci. 101:5082-5091. https://doi.org/10.3168/jds.2017-13607.

Kennedy, K. M., and M. S. Allen. 2019. Hepatic metabolism of propionate relative to meals for cows in the postpartum period. J. Dairy Sci. 102:7997-8010. https://doi.org/10.3168/jds.2018-15907.

Kind, T., G. Wohlgemuth, D. Y. Lee, Y. Lu, M. Palazoglu, S. Shahbaz, and O. Fiehn. 2009. FiehnLib: mass spectral and retention index libraries for metabolomics based on quadrupole and timeof-flight gas chromatography/mass spectrometry. Anal. Chem. 81:10038-10048. https://doi.org/10.1021/ac9019522.

Lemosquet, S., G. Raggio, G. Lobley, H. Rulquin, J. Guinard-Flament, and H. Lapierre. 2009. Whole-body glucose metabolism and mammary energetic nutrient metabolism in lactating dairy cows receiving digestive infusions of casein and propionic acid. J. Dairy Sci. 92:6068-6082. https://doi.org/10.3168/jds.2009-2018.

Leuvenink, H. G., E. J. Bleumer, L. J. Bongers, J. Van Bruchem, and D. Van Der Heide. 1997. Effect of short-term propionate infusion on feed intake and blood parameters in sheep. Am. J. Physiol. 272(6 Pt 1):E997-1001. https://doi.org/10.1152/ajpendo.1997.272 .6.E997.

Maldini, G., and M. S. Allen. 2018. Temporal effects of ruminal propionic acid infusion on feeding behavior of Holstein cows in the postpartum period. J. Dairy Sci. 101:3077-3084. https://doi.org/ 10.3168 /jds.2017-13857.

NRC. 2001. Nutrient Requirements of Dairy Cattle. 7th rev. ed. Natl. Acad. Press, Washington, DC.

Oba, M., and M. S. Allen. 2003. Dose-response effects of intraruminal infusion of propionate on feeding behavior of lactating cows in early or mid lactation. J. Dairy Sci. 86:2922-2931. https://doi.org/ 10.3168/jds.S0022-0302(03)73889-2.

Piantoni, P., C. M. Ylioja, and M. S. Allen. 2015. Feed intake is related to changes in plasma nonesterified fatty acid concentration and hepatic acetyl CoA content following feeding in lactating dairy cows. J. Dairy Sci. 98:6839-6847. https://doi.org/10.3168/jds.2014 -9085 .

Sadri, H., H. R. Rahmani, M. Khorvash, G. R. Ghorbani, and R. M. Bruckmaier. 2012. Chromium supplementation and substitution of barley grain with corn: Effects on metabolite and hormonal responses in periparturient dairy cows. J. Anim. Physiol. Anim. Nutr. (Berl.) 96:220-227. https://doi.org/10.1111/j.1439-0396 .2011.01141.x.

Stocks, S. E., and M. S. Allen. 2012. Hypophagic effects of propionate increase with elevated hepatic acetyl coenzyme A concentration for cows in the early postpartum period. J. Dairy Sci. 95:32593268. https://doi.org/10.3168/jds.2011-4991.

Stocks, S. E., and M. S. Allen. 2013. Hypophagic effects of propionic acid are not attenuated during a 3-day infusion in the early postpartum period in Holstein cows. J. Dairy Sci. 96:4615-4623. https: //doi.org/10.3168/jds.2013-6653.

White, H. 2015. The role of TCA cycle anaplerosis in ketosis and fatty liver in periparturient dairy cows. Animals (Basel) 5:793-802. https://doi.org/10.3390/ani5030384. 